\title{
Design of a Two Input Buck converter (TIBuck) for a Visible Light Communication LED driver based on splitting the power
}

\author{
Daniel G. Aller ${ }^{1}$, Diego G. Lamar ${ }^{1}$, Manuel Arias ${ }^{1}$, Juan Rodríguez ${ }^{2}$, \\ Pablo F. Miaja ${ }^{1}$, and Javier Sebastián ${ }^{1}$ \\ ${ }^{1}$ Electrical, Electronic, Computers and Systems Engineering Department, University of Oviedo, Gijón, Spain. \\ ${ }^{2}$ Centro de Electrónica Industrial (CEI),Universidad Politécnica de Madrid (UPM), Madrid, Spain.
}

\section{Abstract:}

This work proposes a modification of the Two Input Buck converter (TIBuck) working as a high efficiency driver for LEDs and transmitter for Visible Light Communication (VLC). Due to the lower harmonic components and lower voltage stress over the switches, the TIBuck converter is a suitable option for high frequency and fast response converter. The TIBuck converter outperforms the conventional buck converter in terms of efficiency and communication capability because of its lower switching losses and less filtering action required. The second voltage source needed by the TIBuck converter is provided by means of an auxiliary Buck converter, which also implements the average current control over the LEDs. The efficiency of the converter is improved by reducing the power that is been processed at high frequency. Most of the power is processed by an auxiliary Buck converter with lower switching frequency. As experimental results, a TIBuck converter is built based on a low frequency and a high frequency Buck converters connected in parallel regarding the load. The topology reaches a $94 \%$ of efficiency reproducing a 64-QAM with a bitrate up to 1.5 Mbps.

\section{INTRODUCTION}

Nowadays, the majority of the wireless networks are based on the Radio frequency (RF) spectrum, such as in

This work was supported in part by the European Regional Development Fund grants, in part by the Spanish Government under the project MINECO-17-DPI2016-75760-R and RTI2018-099682-AI00, and in part by the Principality of Asturias under the project IDI/2018/000179 and under the scholarship BP17-91.

Email: garciaadaniel@uniovi.es

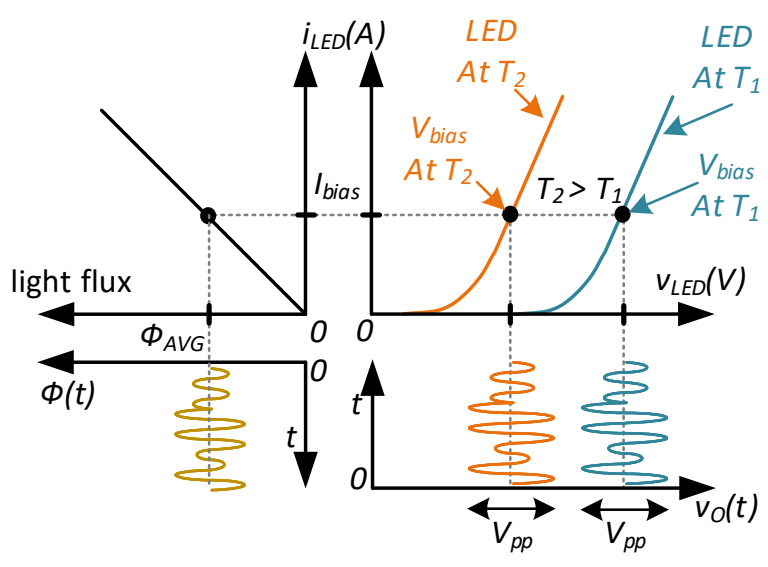

Fig. 1: Temperature effects on the LED curves.

WiFi, Bluethooth, 5G, etc. The projected increase of the wireless traffic is leading to a more strictly regulation and a high congestion of the RF spectrum [1], meaning that new alternatives have to be searched.

One of the solutions that has been proposed in the recent years is Visible Light Communication (VLC) as an alternative for the RF spectrum. It is specially suitable for those environments or applications where the light is already present, creating a more suitable way of communication [2]-[4], or in environments where the RF spectrum is limited or is not a viable option (aviation, hospitals, etc). VLC takes advantage of the widespread use of LED in Solid-State Lighting (SSL), in which the LED capability of fast changes of the emitted light can be used for communication.

In order to use an LED as a VLC transmitter, the circuitry needs to fulfill two tasks: biasing the LEDs to 
generate the adequate lighting level and generating the communication signal. The main task of the LED driver is to maintain the desired bias point, which defines the illumination level of the LED. This is achieved by controlling the average current through the LED regardless of its temperature, as it can be seen in the figure 1. Figure 1 depicts the LED voltage vs current and current vs light flux for two different temperatures $T_{1}$ and $T_{2}$, being $T_{2}$ greater than $T_{1}$. Due to the threshold voltage shift made by the increase of the temperature from $T_{1}$ to $T_{2}$ on the LED, the V vs I curve of the LED is shifted to lower voltages. Due to this effect, the driver has to control the average current in order to always be working on the middle of the linear region of the LED, maximizing the range for the communication signal. Even though there is a temperature effect on the threshold voltage, the slope of the curve is kept unmodified regardless of the temperature, so the maximum peak-to-peak value of signal $V_{p p}$ is kept constant. The maximum $V_{p p}$ in an LED is constrained by the knee region of the LED and the maximum voltage and current. Within that region, the relationship between voltage-current and light can be consider linear. Some types of pre and post equalization have been proposed to reach higher peak to peak values when the LED works outside the linear region [5], [6].

One of the VLC transmitter topologies that has been proposed is based on the use of a regular LED driver for the biasing task (delivering $V_{\text {bias }}$ and controlling $I_{\text {bias }}$ ) connected in parallel with a linear amplifier (i.e. class A or B) [7], [8]. This solution leads to simple implementation with high bit rate but a low efficiency due to the use of a linear amplifier with a theoretical maximum efficiency of $50 \%$ and $78 \%$ respectively but much worse when a high bit rate and more complex modulation is used.

On the other hand, some other proposals are based on adapting the LED driving stage to do both tasks. The use of high frequency DC-DC converters with fast response [9]-[13] has been proposed as a promising alternative to the use of linear amplifiers, achieving high efficiency, around $90 \%$ at high bit rates. One of the disadvantages of using the same converter for bias and communication task is that the biasing power is procesed at high frequency. In a VLC system, the biasing power is much higher than the communication power (around $3 / 4$ of the power is for biasing when $1 / 4$ is for the communication signal) and there is no need to use a high frequency converter for the biasing process because the biasing process only has to control the slow temperature effects over the LED. Then, one way to improve further the efficiency in the converters is by splitting the power [14]. In this case, two converters are used: one low frequency for the biasing and average current control and a high frequency converter for the communication signal, but two input voltages are necessary, one for each converter, which adds complexity to the design of the complete system.

In this paper, a modification of the Two Input Buck converter (TIBuck) for VLC applications based on the idea of splitting the power is presented. The TIBuck converter is implemented with a close loop low frequency converter with a switching frequency of $100 \mathrm{kHz}$ that controls the average current though the LEDs and provides the auxiliary voltages necessary for the TIBuck, and a high frequency TIbuck converter with a switching frequency of $10 \mathrm{MHz}$, delivering the communication signal and working in open loop. The improvement of the efficiency is achieved by delivering most of the power by the low frequency converter and by taking advantage of the reduced switching losses of the TIBuck converter. The topology is able to keep a high efficiency even at high frequency $(10 \mathrm{MHz})$. The communication capability is tested using a 64-QAM digital modulation, reaching a bit rate of $1.5 \mathrm{Mbps}$ with an overall efficiency of $94 \%$.

\section{USE OF THE TIBUCK CONVERTER IN VLC}

The TIBuck converter was originally proposed in [15] as a postregulator. The schematic is depicted in the figure 2.

The TIBuck converter is a modification of the Buck converter where the diode $D_{T I}$ is connected to an auxiliary voltage source $V_{2}$ instead of directly to the negative terminal of the input voltage $V_{1}$. By doing this, the voltage in the input of the filter $v_{s}$ varies from $V_{1}$ to $V_{2}$ as it is depicted in figure 3 . As advantages, the voltage stress over the switches is decreased from $V_{1}$ to $\left(V_{1}-V_{2}\right)$, the harmonic components of the square wave $v_{s}$ are also reduced and the duty cycle resolution is increased. A TIBuck converter has a higher resolution comparing to the Buck converter because for the same increment on the duty cycle, the increment on the output voltage is smaller. On the other hand, the disadvantages are that an auxiliary voltage $V_{2}$ is necessary and the output voltage range is reduced, since the output voltage $v_{o}$ can only vary from $V_{1}$ to $V_{2}$ as it can be seen in 3 .

The latter disadvantage is not really a limitation in the case of a LED lighting or VLC transmitters since the load is an LED string and the output voltage swing necessary is limited by the threshold voltage of the LED and the maximum absolute voltage. If $V_{1}$ is the maximum 


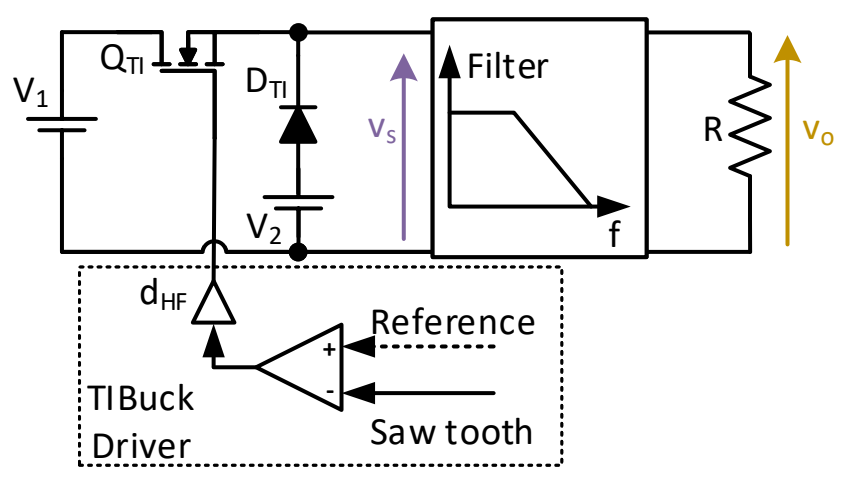

Fig. 2: Schematic of the TIBuck converter.

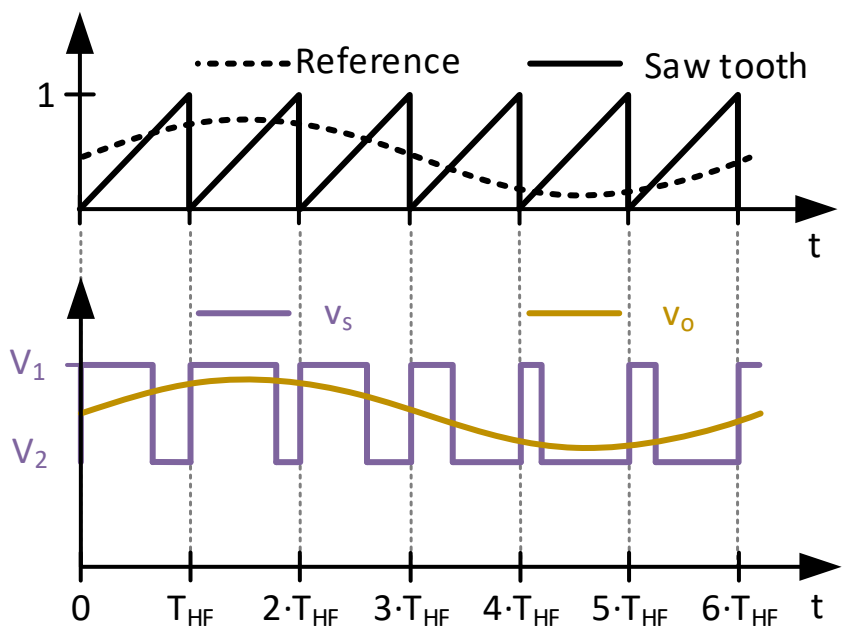

Fig. 3: Switching node voltage $V_{s}$ and output voltage $V_{o}$ in a TIBuck converter using a variable reference.

voltage allowable across the LED and $V_{2}$ is kept below and close to the threshold voltage of the LED, the whole dynamic range necessary for the LED is available as well as the maximum resolution on the duty cycle [16], which is a key factor regarding the communication capability. In modern PWM modulators implemented in DPS or FPGA, the saw tooth represented in 3 is done digitally by a counter, and the reference is a number that is compared to the counter. To reach a resolution of $n$ different duty cycles, the frequency of the clock $f_{C L K}$ has to be $n$ times higher than the frequency of the PWM. In the case of converter for high-frequency DC-DC converters, the switching frequency can be up to $10-20 \mathrm{MHz}$, making the $f_{C L K}$ required impracticably high. By using the TIBuck topology, the available resolution provide by the digital circuitry can be within the voltage range required for LED driving.

Another main factor is to fit the maximum communication signal within the dynamic range of the LED in order to use the whole light amplitude variation on the LED for communication. If the communication signal is fitted inside the duty cycle variation, the output voltage would reproduce the communication signal within the the LED range since $V_{1}$ and $V_{2}$ are designed according to the LED range. To do this, since the communication signal has 0 average value and positive and negative parts, an offset over the reference is necessary, so the duty cycle $d_{H F}$ of the TIBuck has an average value $D_{H F}$ due to the offset and a variation $\Delta d_{H F}$ proportional to the communication signal, depicted in 3 as a sinusoidal reference signal, but in reality it would be a much more complex communication signal. If the offset $D_{H F}$ is set to 0.5 , the maximum variation $\Delta d_{H F}$, also 0.5 , is achieved. The output signal can be expressed in terms of the duty cycle $d_{H F}$ and the input voltages $V_{1}$ and $V_{2}$ as follow.

$v_{o}=d_{H F}\left(V_{1}-V_{2}\right)+V_{2} \leftrightarrow v_{o}=\left(D_{H F}+\Delta d_{H F}\right)\left(V_{1}-V_{2}\right)+V_{2}$

The decrease of the switching losses, less harmonic components and a higher duty cycle resolution make the TIBuck suitable for high frequency and fast response conversion [17]-[19]. With a proper design of the filter, the reference signal can be modulated into the PWM $\left(\Delta d_{H F}\right)$, provided that the frequency of the reference is lower than the switching frequency and the filter allows the reference frequency to pass through, but the frequencies of the PWM harmonic components are filtered [20]. This filtering process can be also done in a normal buck converter and it also depends on how selective the filter is, but on the TIBuck converter the harmonic components are lower, therefore the filtering process is less demanding, meaning either needing lower order in the filter or higher cut-off frequency.

\section{A. Modification of the TIBuck converter for VLC LED driving}

The proposed modification of the TIBuck converter for VLC can be seen in the figure 4. Since two input voltages $V_{1}$ and $V_{2}$ are necessary and $V_{2}<V_{1}, V_{2}$ can be obtained from $V_{1}$ by adding an auxiliary Buck converter in between. $d_{L F}$ is the duty cycle of the converter that generates $V_{2}$, where

$$
V_{2}=d_{L F} V_{1}
$$

and $d_{H F}$ is the duty of the TIBuck converter. By replacing (2) into (1), the output voltage can be expressed in terms of the duty of both converters as

$$
v_{o}=d_{H F}\left(V_{1}-d_{L F} V_{1}\right)+d_{L F} V_{1} .
$$




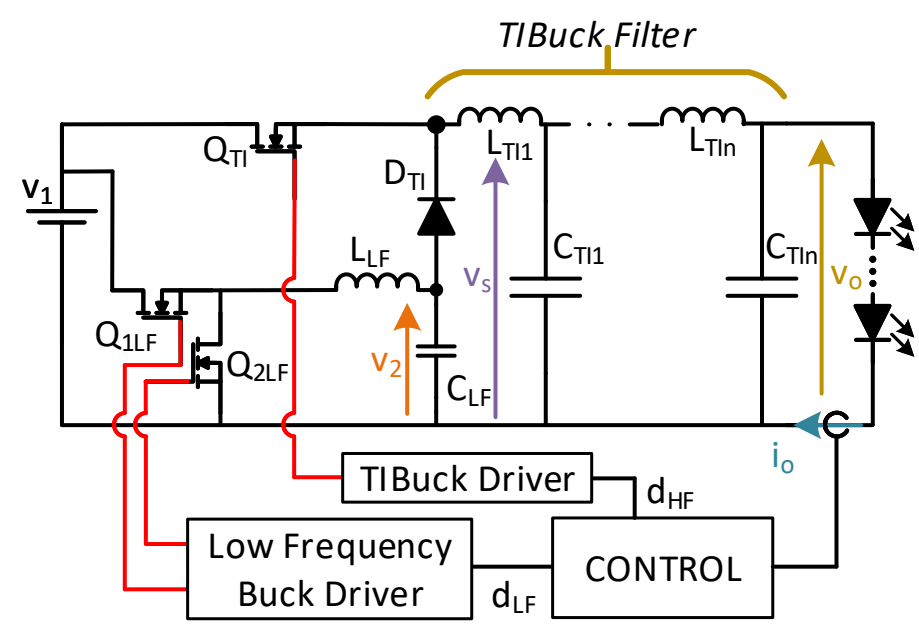

Fig. 4: Modification of the TIBuck converter by adding an auxiliary converter with current control.

Assuming that the switching frequency of the additional Buck converter is much lower than the TIBuck converter, the duty of the TIBuck during a switching period of the Buck can be replaced by its average value $D_{H F}$, which by design is equal to 0.5 , giving

$$
v_{o}=0.5\left(1+d_{L F}\right) V_{1} .
$$

According to (4), the control on the average voltage through the LEDs (and, therefore, the average current across and light emitted by them) can be implemented throughout $d_{L F}$ because there is a linear relation between the duty cycle and the average output voltage. On the other hand, because of the switching frequency of the TIBuck is much higher and during a switching period of the TIBuck, $V_{1}$ and $V_{2}$ are kept constant, meaning that the instantaneous value of the output voltage is controlled by $d_{H F}$ according to (1).

As a summary, the low frequency Buck converter works in close loop controlling the average current though the LEDs while the High Frequency TIBuck converter works in open loop and it delivers the communication signal.

\section{EXPERIMENTAL RESULTS}

As experimental results, a modified TIBuck converter adapted for VLC is built. The design is based on two converters: a low frequency Buck converter which generates the auxiliary voltage $V_{2}$ necessary for the TIBuck converter and also implements the average current control over the LED (close loop); and the high frequency TIBuck converter that generates the communication signal (open loop). The load is a string of 8
HB-LEDs XLamp $M X-3$. The input voltage is $28 \mathrm{~V}$. As it is mentioned before, the input voltage is designed to be close to the maximum voltage of the LED string to reach the maximum resolution on the duty cycle on the TIBuck converter. The average current through the LEDs is kept at $0.25 \mathrm{~A}$ (half of the maximum current). The output power is around $8 \mathrm{~W}$ achieving an overall efficiency of $94 \%$. The efficiency is measured by getting the RMS input and output power, which is specially critical regarding the output power since the communication power has to be taken into account. The output voltage and current are taken using an oscilloscope and post processing to calculate the RMS output power.

\section{A. Low Frequency Buck design}

The low frequency Buck is designed with a switching frequency of $100 \mathrm{kHz}$, using a 2nd order low-pass filter with a cutoff frequency of $10 \mathrm{kHz}$, one decade below. The output filter values are shown in the table I. A trade off on the selection of the switching frequency has to be defined. On one had, the higher the frequency, the higher the switching losses in the converter, and since this converter is delivering most of the power, the performance of this converter has a big impact on the overall efficiency. On the other hand, the lower the frequency, the lower the switching losses but the greater the components of the filter. Also an advantage of decreasing the switching frequency is the noise added to the communication signal. Any ripple on the voltage $V_{2}$ is seen by the LEDs and also added to the communication signal. This ripple acts as a noise in the communication signal.

Table I: COMPONENT VALUE OF THE 2ND ORDER LOW PASS FILTER FOR THE LOW FREQUENCY BUCK CONVERTER

\begin{tabular}{cc}
\hline$L_{L F}$ & $C_{L F}$ \\
\hline \hline $49 \mu H$ & $9 \mu F$ \\
\hline
\end{tabular}

The converter works in close loop and the control is implemented in a FPGA. The current is measured by an $\mathrm{ADC}$, and a digital filter eliminates the communication components on the current since only the average current thought the LED has to be controlled. A digital PI controller is used with a bandwidth of $10 \mathrm{~Hz}$. The temperature drift on the LED has a slow dynamic there is no need for a high speed controller.

Also no further considerations about the plant of the converter are needed since the switching frequencies of the low frequency Buck converter is much lower than the switching frequency of the TIBuck converter. The load seen by the low frequency Buck depends on 
the number of LEDs (constant) and the average duty cycle of the TIBuck converter, which by design is also constant, meaning that regarding the control design, the low frequency Buck has the same dynamic behavior as a normal Buck converter.

For the component selection, a dual MOSFET CSD88539 is chosen for the MOSFETs $Q_{1 L F}$ and $Q_{2 L F}$ and a half bridge driver ISL6700 is used to drive them.

\section{B. Communication scheme}

The converter reproduces a 64-QAM modulation, with a carrier frequency of $1 \mathrm{MHz}$, a symbol period of 4 signal periods, achieving a maximum bit rate of $1.5 \mathrm{Mbps}$.

\section{High Frequency TIBuck design}

The TIBuck converter has to be able to reproduce a 1 $\mathrm{MHz}$ signal with low distortion and enough resolution. The switching frequency and the filter are designed according to the modulation scheme. The TIBuck converter is designed with a switching frequency of $10 \mathrm{MHz}$, one decade higher than the carrier frequency of the modulation. A trade off between resolution, switching losses and filter order has to be defined. The higher the switching frequency, the lower the resolution and the higher the switching losses, but the filtering action would be less demanding, so a filter with lower order would be necessary. On the other hand, with a lower switching frequency, the resolution would be higher and the power losses would be lower but if the switching frequency becomes closer to the carrier frequency of the modulation, the order of the filter needs to be higher in order to separate the communication spectrum from the PWM spectrum [11], [20].

The filter used is a 6th order low-pass filter with a cutoff frequency of $2.5 \mathrm{MHz}$. The component values are shown in the table II.

For the component selection, a RF MOSFET PD84010S-E as $Q_{T I}$ and a diode UPS115UE3 as $D_{T I}$ are used. Due to the high switching frequency, a fast driver EL7155CSZ and a digital isolator ISO721 are used to drive the transistor $Q_{T I}$.

Table II: COMPONENT VALUE OF THE 6TH ORDER LOW PASS FILTER FOR THE HIGH FREQUENCY TIBUCK CONVERTER

\begin{tabular}{cccccc}
\hline$L_{T I 1}$ & $C_{T I 1}$ & $L_{T I 2}$ & $C_{T I 2}$ & $L_{T I 3}$ & $C_{T I 3}$ \\
\hline \hline $1.7 \mu H$ & $9.9 \mu F$ & $2.2 \mu H$ & $9.9 \mu F$ & $1.9 \mu H$ & $5.72 \mu F$ \\
\hline
\end{tabular}

Figure 5 shows the most representative waveforms of the TIBuck reproducing the communication signal. $V_{o}$ and $I_{o}$ are the voltage across and the current through the LED string. The average current is kept constant by the

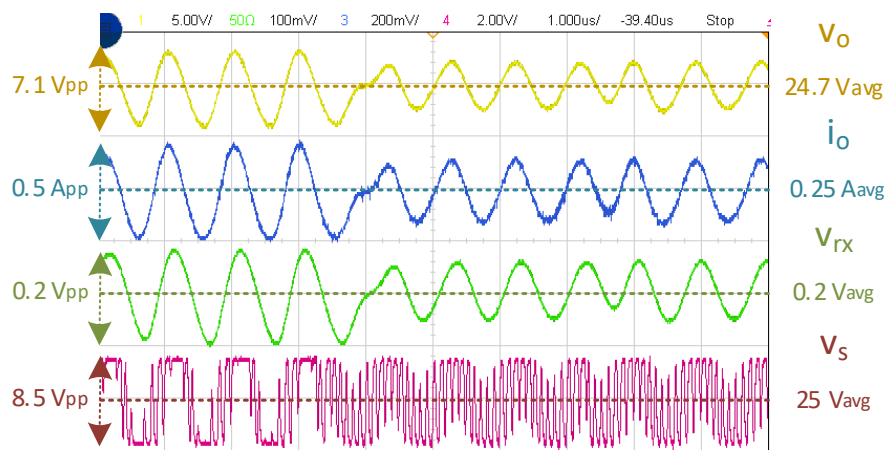

Fig. 5: Output voltage $V_{o}$, output current $I_{o}$, voltage on the optical receiver $V_{r x}$ and the switching node voltage $V_{s}$ on the TIBuck converter.

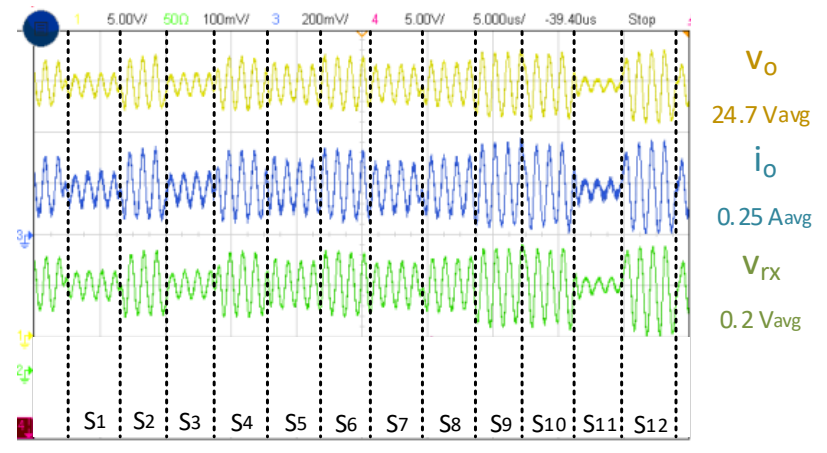

Fig. 6: Output voltage $V_{o}$, output current $I_{o}$ and voltage on the optical receiver $V_{r x}$ during the transmission of 12 symbols.

current controller while the instantaneous value follows the communication signal, reproducing the symbols of the modulation. $V_{s}$ is the switching node of the TIBuck converter showing a square waveform varying from $V_{1}=$ $28 \mathrm{~V}$ to $V_{2}=20 \mathrm{~V}$. The value of $V_{2}$ is controlled by the low frequency Buck according to the average value of $I_{o} . V_{r x}$ is the light received by a optical receiver placed in from of the LEDs.

Figure 6 shows a longer transmition where 12 different symbols are being transmitted during the communication. The converter is able to reproduce the variations of the amplitude and phase necessary for the communication, as it can be seen in the voltage $v_{o}$ and the current $i_{o}$. Also $V_{r x}$ is obtained by the optical receiver. It can be seen that the light reproduces the amplitude and phase changes for each symbol that is being sent.

\section{Conclusions}

As conclusions, the design of a TIBuck converter for LED driving working as a VLC transmitter is presented. The original topology is slightly modified in order to only use one input voltage by adding an additional buck converter, which also implements the current loop 
control for the average LED current. The idea is based on splitting of the power, meaning that the majority of the power is delivered at low frequency (the biasing power), and the power delivered at high frequency is reduced. As well as the power, the voltage stress over the high frequency switches is reduced comparing to the conventional Buck converter, which also increases the efficiency. The experimental results show an efficiency of $94 \%$ reproducing a 64-QAM digital modulation, with a bit rate of 1.5 Mbps.

\section{REFERENCES}

[1] Cisco Systems. (2017) Cisco visual networking index: Global mobile data traffic forecast update, 2016-2021.

[2] "Ieee standard for local and metropolitan area networks-part 15.7: Short-range wireless optical communication using visible light," IEEE Std 802.15.7-2011, pp. 1-309, Sept 2011.

[3] A. Jovicic, J. Li, and T. Richardson, "Visible light communication: opportunities, challenges and the path to market," IEEE Communications Magazine, vol. 51, no. 12, pp. 26-32, December 2013.

[4] H. Elgala, R. Mesleh, and H. Haas, "Indoor optical wireless communication: potential and state-of-the-art," IEEE Communications Magazine, vol. 49, no. 9, pp. 56-62, September 2011.

[5] R. Mitra and V. Bhatia, "Low complexity post-distorter for visible light communications," IEEE Communications Letters, vol. 7798, no. c, pp. 1-1, 2017. [Online]. Available: http://ieeexplore.ieee.org/document/7942133/

[6] X. Li, H. Chen, S. Li, Q. Gao, C. Gong, and Z. Xu, "Volterrabased Nonlinear Equalization for Nonlinearity Mitigation in Organic VLC," pp. 616-621, 2017.

[7] H. Chun, S. Rajbhandari, G. Faulkner, D. Tsonev, E. Xie, J. J. D. McKendry, E. Gu, M. D. Dawson, D. C. O'Brien, and H. Haas, "Led based wavelength division multiplexed $10 \mathrm{gb} / \mathrm{s}$ visible light communications," Journal of Lightwave Technology, vol. 34, no. 13, pp. 3047-3052, July 2016.

[8] J. Vučić, C. Kottke, K. Habel, and K. D. Langer, "803 mbit/s visible light wdm link based on dmt modulation of a single rgb led luminary," in 2011 Optical Fiber Communication Conference and Exposition and the National Fiber Optic Engineers Conference, March 2011, pp. 1-3.

[9] J. Rodriguez, D. G. Lamar, P. F. Miaja, and J. Sebastian, "Reproducing Single-Carrier Digital Modulation Schemes for VLC by Controlling the First Switching Harmonic of the DC-DC Power Converter Output Voltage Ripple," IEEE Transactions on Power Electronics, vol. 33, no. 9, pp. 7994-8010, Sept 2018.

[10] J. Sebastian, D. G. Lamar, D. G. Aller, J. Rodriguez, and P. F. Miaja, "On the Role of Power Electronics in Visible Light Communication," IEEE Journal of Emerging and Selected Topics in Power Electronics, pp. 1-1, 2018.

[11] J. Rodriguez, D. G. Lamar, D. G. Aller, P. F. Miaja, and J. Sebastian, "Efficient Visible Light Communication Transmitters Based on Switching-Mode dc-dc Converters," Sensors, vol. 18, no. 4, 2018.

[12] J. Rodríguez Mendez, D. G. Lamar, D. G. Aller, P. F. Miaja, and J. Sebastián, "Reproducing multicarrier modulation schemes for visible light communication with the ripple modulation technique," IEEE Transactions on Industrial Electronics, vol. 67, no. 2, pp. 1532-1543, Feb 2020.
[13] F. Loose, L. Teixeira, R. R. Duarte, M. A. Dalla Costa, and C. H. Barriquello, "On the Use of the Intrinsic Ripple of a Buck Converter for Visible Light Communication in LED Drivers," IEEE Journal of Emerging and Selected Topics in Power Electronics, vol. 6, no. 3, pp. 1235-1245, Sep. 2018.

[14] J. Rodriguez, D. G. Lamar, P. F. Miaja, D. G. Aller, and J. Sebastian, "Power Efficient VLC Transmitter Based on PulseWidth Modulated DC-DC Converters and the Split of the Power," IEEE Transactions on Power Electronics, pp. 1-1, 2018.

[15] J. Sebastian, P. J. Villegas, F. Nuno, and M. M. Hernando, "High-efficiency and wide-bandwidth performance obtainable from a two-input buck converter," IEEE Transactions on Power Electronics, vol. 13, no. 4, pp. 706-717, July 1998.

[16] M. Arias, D. G. Lamar, J. Sebastian, D. Balocco, and A. A. Diallo, "High-efficiency LED driver without electrolytic capacitor for street lighting," IEEE Transactions on Industry Applications, vol. 49, no. 1, pp. 127-137, 2013.

[17] V. Yousefzadeh, E. Alarcon, and D. Maksimovic, "Threelevel buck converter for envelope tracking applications," IEEE Transactions on Power Electronics, vol. 21, no. 2, pp. 549-552, March 2006.

[18] M. Rodríguez, P. Fernández-Miaja, A. Rodríguez, and J. Sebastián, "A multiple-input digitally controlled buck converter for envelope tracking applications in radiofrequency power amplifiers," IEEE Transactions on Power Electronics, vol. 25, no. 2, pp. 369-381, 2010.

[19] P. F. Miaja, A. Rodriguez, J. Sebastian, and M. Rodriguez, "Enhancements of the multiple input buck converter used for Envelope Tracking applications by improved output filter design and multiphase operation," 2012 IEEE Energy Conversion Congress and Exposition, ECCE 2012, pp. 1841-1848, 2012.

[20] J. Sebastián, P. Fernández-Miaja, F. J. Ortega-González, M. Patiño, and M. Rodríguez, "Design of a Two-Phase Buck Converter With Fourth-Order Output Filter for Envelope Amplifiers of Limited Bandwidth," IEEE Transactions on Power Electronics, vol. 29, no. 11, pp. 5933-5948, Nov 2014. 